Significant inter-observer variation in the diagnosis of extrapancreatic necrosis and type of pancreatic collections in acute pancreatitis - An international multicenter evaluation of the revised Atlanta classification

\title{
Sternby, Hanna
}

2016

Sternby , H , Verdonk, R C , Aguilar , G , Dimova , A , Ignatavicius , P , Ilzarbe , L , Koiva , P , Lantto , E , Loigom , T , Penttilä , A , Regner , S , Rosendahl , J , Strahinova , V , Zackrisson , S , Zviniene, K \& Bollen , T L 2016, ' Significant inter-observer variation in the diagnosis of extrapancreatic necrosis and type of pancreatic collections in acute pancreatitis - An international multicenter evaluation of the revised Atlanta classification ', Pancreatology , vol. 16 , no. 5 , pp. $791-797$. https://doi.org/10.1016/j.pan.2016.08.007

http://hdl.handle.net/10138/228314

https://doi.org/10.1016/j.pan.2016.08.007

publishedVersion

Downloaded from Helda, University of Helsinki institutional repository.

This is an electronic reprint of the original article.

This reprint may differ from the original in pagination and typographic detail.

Please cite the original version. 
Original Article

\title{
Significant inter-observer variation in the diagnosis of extrapancreatic necrosis and type of pancreatic collections in acute pancreatitis - An international multicenter evaluation of the revised Atlanta classification
}

\author{
Hanna Sternby ${ }^{\text {a, * }}$, Robert C. Verdonk ${ }^{b}$, Guadalupe Aguilar ${ }^{c}$, Alexandra Dimova ${ }^{\text {, }}$, \\ Povilas Ignatavicius ${ }^{\mathrm{e}}$, Lucas Ilzarbe ${ }^{\mathrm{f}}$, Peeter Koiva ${ }^{\mathrm{g}}$, Eila Lantto ${ }^{\mathrm{h}}$, Tonis Loigom ${ }^{\mathrm{i}}$, \\ Anne Penttilä ${ }^{j}$, Sara Regnér ${ }^{a}$, Jonas Rosendahl ${ }^{k}$, Vanya Strahinova ${ }^{l}$, Sophia Zackrisson ${ }^{m}$, \\ Kristina Zviniene ${ }^{\mathrm{n}}$, Thomas L. Bollen ${ }^{\mathrm{O}}$ \\ ${ }^{a}$ Department of Surgery, Institution of Clinical Sciences Malmö, Lund University, Malmö, Sweden \\ b Department of Gastroenterology, St. Antonius Hospital, Nieuwegein, The Netherlands \\ ${ }^{\mathrm{c}}$ Department of Radiology, Hospital del Mar, Barcelona, Spain \\ d Department of Surgery, University Hospital for Emergency Medicine "Pirogov", Sofia, Bulgaria \\ e Department of Surgery, Lithuanian University of Health Sciences, Kaunas, Lithuania \\ f Department of Gastroenterology, Hospital del Mar, Barcelona, Spain \\ ${ }^{g}$ Department of Gastroenterology, East Tallinn Central Hospital, Tallinn, Estonia \\ h Department of Radiology, Helsinki University Central Hospital, Helsinki, Finland \\ ${ }^{\mathrm{i}}$ Department of Radiology, East Tallinn Central Hospital, Tallinn, Estonia \\ ${ }^{\mathrm{j}}$ Department of Surgery, Helsinki University Central Hospital, Helsinki, Finland \\ k Department of Internal Medicine, Neurology and Dermatology, Division of Gastroenterology and Rheumatology, Leipzig, Germany \\ ${ }^{1}$ Department of Emergency Radiology, University Hospital for Emergency Medicine "Pigorov", Sofia, Bulgaria \\ ${ }^{\mathrm{m}}$ Department of Radiology, Institution of Clinical Sciences Malmö, Lund University, Malmö, Sweden \\ ${ }^{\mathrm{n}}$ Department of Radiology, Lithuanian University of Health Sciences, Kaunas, Lithuania \\ ${ }^{\circ}$ Department of Radiology, St. Antonius Hospital, Nieuwegein, The Netherlands
}

\section{A R T I C L E I N F O}

\section{Article history:}

Received 10 February 2016

Received in revised form

14 June 2016

Accepted 11 August 2016

Available online 13 August 2016

\section{Keywords:}

Acute pancreatitis

Classification

Computed tomography

Extrapancreatic necrosis

Necrotising pancreatitis

\begin{abstract}
A B S T R A C T
Background: For consistent reporting and better comparison of data in research the revised Atlanta classification (RAC) proposes new computed tomography (CT) criteria to describe the morphology of acute pancreatitis (AP). The aim of this study was to analyse the interobserver agreement among radiologists in evaluating CT morphology by using the new RAC criteria in patients with AP.

Methods: Patients with a first episode of AP who obtained a CT were identified and consecutively enrolled at six European centres backwards from January 2013 to January 2012. A local radiologist at each center and a central expert radiologist scored the CTs separately using the RAC criteria. Center dependent and independent interobserver agreement was determined using Kappa statistics.

Results: In total, 285 patients with 388 CTs were included. For most CT criteria, interobserver agreement was moderate to substantial. In four categories, the center independent kappa values were fair: extrapancreatic necrosis (EXPN) (0.326), type of pancreatitis (0.370), characteristics of collections (0.408), and appropriate term of collections (0.356). The fair kappa values relate to discrepancies in the identification of extrapancreatic necrotic material. The local radiologists diagnosed EXPN (33\% versus 59\%, $P<0.0001$ ) and non-homogeneous collections (35\% versus $66 \%, P<0.0001$ ) significantly less frequent than the central expert. Cases read by the central expert showed superior correlation with clinical outcome.
\end{abstract}

Abbreviations: AP, acute pancreatitis; CECT, contrast-enhanced computed tomography; Central exp, Central expert; CRP, C-reactive protein; CT, computed tomography;

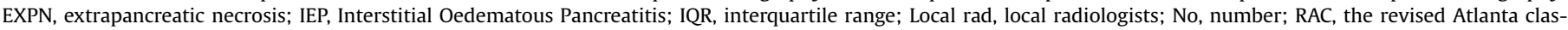
sification; SIRS, systemic inflammatory response syndrome.

* Corresponding author. Department of Surgery, Skåne University Hospital, Lund University, Jan Waldenströms gata 44,20502 Malmö, Sweden. Tel.: +46 736 297981.

E-mail address: Hanna.Sternby@med.lu.se (H. Sternby). 
Conclusion: Diagnosis of EXPN and recognition of non-homogeneous collections show only fair agreement potentially resulting in inconsistent reporting of morphologic findings.

๑) 2016 IAP and EPC. Published by Elsevier B.V. All rights reserved.

\section{Introduction}

Acute pancreatitis (AP) is a complex disease with potentially severe and fatal outcome [1,2]. Simple but clear definitions of the disease are crucial in interdisciplinary consultation, communication, and in reporting of clinical research. Such were the incentives to update the 1992 Atlanta Classification on AP [1]. Besides redefining the disease into three levels of clinical severity, the 2012 revised Atlanta Classification (RAC) has put substantial efforts into clarifying the terminology on the morphologic subtypes of AP and associated peripancreatic collections based on computed tomography (CT)-based criteria [1]. Two morphologic types of AP are discriminated: acute interstitial oedematous pancreatitis and acute necrotising pancreatitis. Acute necrotising pancreatitis is subdivided into three forms: pancreatic parenchymal necrosis, extrapancreatic necrosis (EXPN), and combined necrosis. Peripancreatic collections are classified into four types depending on content and maturation. Acute peripancreatic fluid collections and pancreatic pseudocysts are composed of fluid only and occur in interstitial oedematous pancreatitis. On CT, these collections show a homogeneous fluid density with no or incomplete well-defined wall (acute peripancreatic fluid collection) or a complete wall (pseudocyst). Acute necrotic collections and walled-off necrosis are associated with acute necrotising pancreatitis and contain varying amounts of fluid and necrotic material. On CT, these collections have various densities (fat, fluid, solid material) with no or incomplete well-defined wall (acute necrotic collection) or a complete wall (walled-off necrosis) [1,3-5]. The RAC provides approximate time frames for these pancreatic collections. Acute peripancreatic fluid collection and acute necrotic collection pertain to the first four weeks of disease after which they usually turn into a completely encapsulated pseudocyst and walled-off necrosis, respectively.

It is well established that the morphologic types of AP differ in outcomes, therapies, and prognosis. For prognostication, stratification, and comparing of interinstitutional data, accurate assessment of AP morphology in the different stages of disease is imperative [1]. The extent of variation in interpretation of the new CT criteria is, however, unknown [6-8]. The aim of this study was to assess the interobserver agreement among radiologists in the evaluation of CT morphology using the RAC criteria.

\section{Methods}

\subsection{Patients and study design}

Patients $>18$ years with a first episode of AP were consecutively identified at six European study centres, going backwards from January 2013 to January 2012. Each center included 50 patients in whom at least one contrast-enhanced CT (CECT) was performed. The cases were anonymously enrolled and each patient obtained a code blinded for all investigators except for the referring center. CECTs performed within 3 months from date of admission were recorded and subsequently reviewed and scored by a local radiologist at each center. The time frame of 3 months was chosen because most CTs are performed within this period and controversies in nomenclature and management of pancreatic collections are most evident during this phase. Exclusion criteria were insufficient quality of the CECT, signs of chronic pancreatitis (i.e. pancreatic calcifications) or patients with prior pancreatitis-related invasive intervention, except from endoscopic retrograde cholangiography. Each CECT was performed in the pancreatic and/or in the portal venous phase (see Supplementary file 1 for CT specifications). Severity and CT morphology of AP were defined according to the RAC (see Box 1 for definitions) [1].

The following clinical data was collected from review of medical notes: systemic inflammatory response syndrome (SIRS) upon admission, highest level of C-reactive protein (CRP) during hospitalisation, need for invasive intervention, organ failure (persistent and transient, in line with the RAC), and in-hospital mortality. The six participating local radiologists had expertise in the field of abdominal radiology, each with more than five years' experience. A short instruction sheet was provided to local radiologists to assist in interpretation (Supplementary file 2). All individual CECTs were scored according to a protocol based on the parameters stated in the RAC (Supplementary file 3). Subsequently, all CECTs were reviewed and scored (using the same scoring sheet) by a central expert radiologist (T.L.B) using open source DICOM viewer software (32-bit OsiriX version 3.3, Geneva, Switzerland). Local and central reviewers were blinded to any clinical data except for the timing (number of days after onset of symptoms) of each CECT. Formal approval of the local medical ethical committee was requested and obtained at each study center.

\section{Box 1}

Morphological features and CECT criteria in AP according to the RAC.

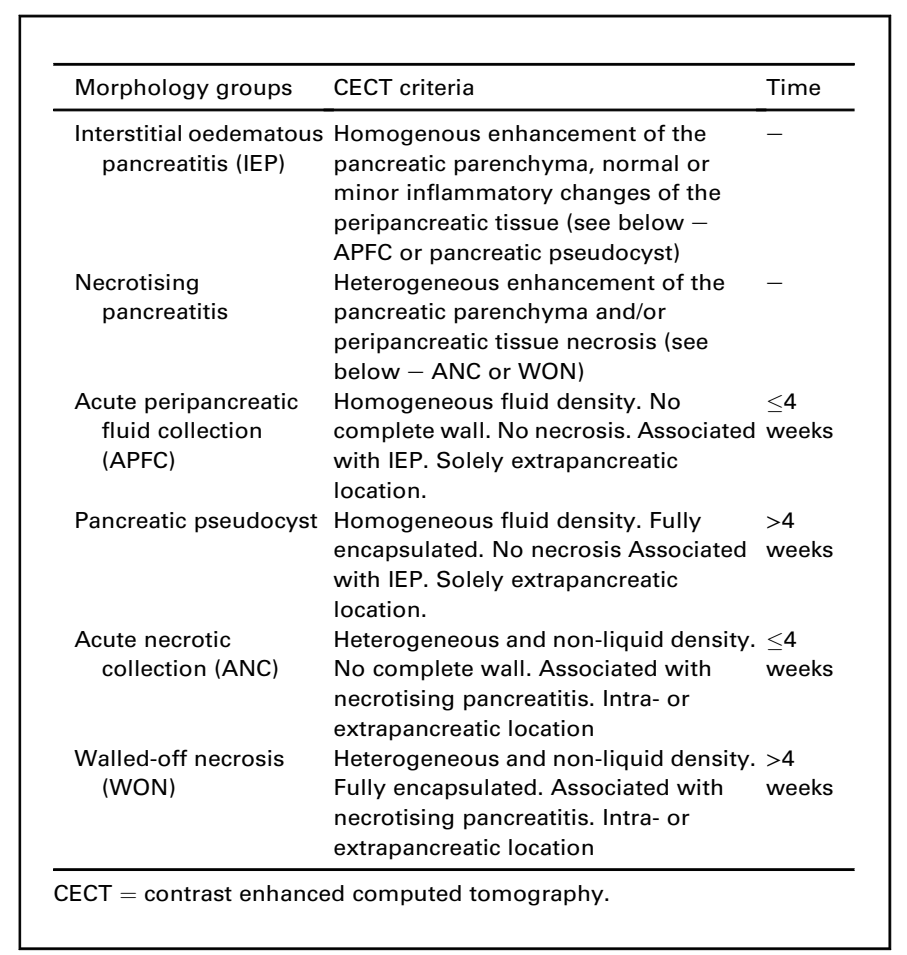




\subsection{Statistical analysis}

Sample size calculations for variables with 2, 3, and 4 categories were performed because most of the variables of the revised Atlanta criteria are based on 2, 3, or 4 categories. Kappa values of $>0.40$ and $>0.60$ were used since they represent at least moderate and substantial agreement [9]. Based on such a calculation, in a test for agreement between two raters, a sample size of 360 CECTs would provide a $95 \%$ confidence interval for the $\kappa$ statistic with a width not greater than 0.20 . Assuming that several patients would have more than one CECT performed, a total of 50 patients were included per center. Interobserver agreement was calculated between the local and the central radiologist, using Cohen's kappa test, for each of the categories scored on the radiology sheet. Agreement levels were defined as: $\kappa$ level $0.00-0.20$ slight; $0.21-0.40$ fair; $0.41-0.60$ moderate; $0.61-0.80$ substantial; and $0.81-1.00$ almost perfect. Continuous data analysis was conducted using Mann-Whitney $U$ test. Wilcoxon Signed Rank test was used for paired sample analysis. $P<0.05$ was considered statistically significant. All analysis was performed using IBM SPSS Statistics for Windows, version 21 and 22, Armonk, NY:IBM corp.

\section{Results}

\subsection{Patients}

In total, 301 patients were included at six European centres of whom 159 (56\%) were male with a median age of 58 years (range 18-92). Sixteen patients were excluded due to reasons stated in the CECT section below. Baseline characteristics of the remaining 285 patients are summarised in Table 1. Etiology of AP differed substantially between the centres. According to the RAC, 37.5\% of the patients had mild AP, 51.5\% moderately severe AP and 11.0\% severe AP [1]. Overall fourteen patients died (4.9\%, range 0-14\%), whereas mortality within the severe group was 32.3\% (range 0-78\%). Each center admits 175-250 patients with AP annually, except for center F where the figure is approximately 470 .

\subsection{Contrast-enhanced computed tomography}

A total of 405 CECTs derived from 301 patients were collected. Seventeen CECT studies were excluded due to insufficient quality of the CECT or signs of chronic pancreatitis, leaving a study cohort of 285 patients with 388 CECTs. Data on CECTs for the separate centres are presented in Table 2. Median time from onset of disease to CECT for all centres was 7 days (range 0-90, interquartile range 3-13).

\subsection{Interobserver agreement}

For specific information concerning all categories evaluated we refer to Supplementary file 2. The kappa values representing the interobserver agreement are shown in Table 3. There was substantial agreement in seven categories: "Necrosis - Neck" (0.618); "Necrosis - Body" (0.628); "Necrosis - Tail" (0.617); presence of "Collections" (0.756); "Location of Collections" (0.633); presence of "Wall" (0.675); and presence of "Intraluminal Gas and/or Fluid level" (0.764). Moderate agreement was reached on "Parenchymal Necrosis" (0.539) and "Necrosis - Head" (0.516). Finally, there was fair agreement on the categories "Type of Pancreatitis" (0.370), "Extrapancreatic Necrosis" (0.326), "Characteristics of Collection" (0.408), and "Collection - most appropriate term" (0.356). The center dependent kappa values differed considerably between centres.

Discrepancies in the identification of EXPN are shown in Table 4. For image samples see Fig. 1a,b and 2a-c. The expert radiologist diagnosed EXPN significantly more often than the local radiologists (59\% vs. $33 \%, P<0.0001$ ). Table 4 shows that this difference in total number of EXPN stems from the subgroup of isolated EXPN. Since the RAC acknowledges that EXPN might be difficult to diagnose within the first week, interobserver agreement was recalculated for the categories with low kappa values excluding CECTs performed within $72 \mathrm{~h}$, seven days and two weeks after onset of disease (see Supplementary file 4). In this subanalysis, kappa values did improve only for CECTs performed after two weeks.

Morphological findings scored by the central and local radiologists were correlated with clinical outcome parameters (see Table 5). Cases read as interstitial oedematous pancreatitis and isolated EXPN by the central expert correlate significantly better with clinical outcome than scoring by local radiologists. Given the good interobserver agreement for pancreatic parenchymal necrosis, results did not differ significantly between central and local radiologists for this subgroup (Supplementary file 5).

\section{Discussion}

The RAC proposed a new set of morphologic CT-based criteria to account for alleged shortcomings of the 1992 Atlanta classification $[1,2]$. One of the major aims of the RAC was to ease and ensure

Table 1

Baseline characteristics of patients in each center and all centres combined.

\begin{tabular}{|c|c|c|c|c|c|c|c|}
\hline & All patients $\mathrm{n}=285$ & Center B $\mathrm{n}=42$ & Center $\mathrm{C} \mathrm{n}=48$ & Center D $\mathrm{n}=50$ & Center $\mathrm{E} \mathrm{n}=48$ & Center $\mathrm{F} \mathrm{n}=47$ & Center $\mathrm{G} \mathrm{n}=50$ \\
\hline Male sex & $159(56 \%)$ & $28(67 \%)$ & $24(50 \%)$ & $29(58 \%)$ & $18(37 \%)$ & $32(68 \%)$ & $28(68 \%)$ \\
\hline Age (years) & $\begin{array}{l}58(18-92) \mathrm{IQR}(45 \\
-71)\end{array}$ & $\begin{array}{l}46(22-83) \mathrm{IQR} 34 \\
-61\end{array}$ & $\begin{array}{l}65(18-88) \text { IQR } 52 \\
-76\end{array}$ & $\begin{array}{l}62(21-92) \mathrm{IQR} \\
41-76\end{array}$ & $\begin{array}{l}60(22-88) I Q R \\
42-73\end{array}$ & $\begin{array}{l}52(35-85) \mathrm{IQR} \\
45-68\end{array}$ & $\begin{array}{l}62(23-87) \mathrm{IQR} \\
52-73\end{array}$ \\
\hline \multicolumn{8}{|l|}{ Etiology (\%) } \\
\hline Biliary & 36.6 & 24.4 & 56.3 & 18.0 & 54.1 & 19.2 & 46.0 \\
\hline Alcohol & 35.9 & 43.9 & 14.5 & 26.0 & 41.7 & 63.8 & 28.0 \\
\hline Other & 27.5 & 31.7 & 29.2 & 56.0 & 4.2 & 17.0 & 26.0 \\
\hline $\begin{array}{l}\text { Highest CRP } \\
\quad(\mathrm{mg} / \mathrm{l})\end{array}$ & $261(0-553)$ & $259(44-493)$ & $208(3-480)$ & $281(0-477)$ & $258(2-444)$ & $278(9-519)$ & $277(41-553)$ \\
\hline \multicolumn{8}{|c|}{ Classification $\mathrm{n}(\%)$} \\
\hline Mild & $107(37.5)$ & $17(40.5)$ & $26(54.2)$ & $10(20.0)$ & $11(22.9)$ & $19(40.4)$ & $24(48.0)$ \\
\hline $\begin{array}{l}\text { Moderately } \\
\text { severe }\end{array}$ & $147(51.5)$ & $24(57.1)$ & $18(37.5)$ & $31(62.0)$ & 35 (72.9) & $20(42.6)$ & $19(38.0)$ \\
\hline Severe & $31(11.0)$ & $1(2.4)$ & $4(8.3)$ & $9(18.0)$ & $2(4.2)$ & $8(17.0)$ & $7(14.0)$ \\
\hline Mortality (\%) & 4.9 & 2.0 & 0.0 & 14.0 & 0.0 & 8.5 & 4.0 \\
\hline
\end{tabular}

Continuous variables are median. Range and IQR are displayed if applicable.

$\mathrm{n}=$ number of patients.

$\mathrm{IQR}=$ Inter quartile range.

$\mathrm{CRP}=\mathrm{C}$-reactive protein. 
Table 2

Characteristics of CECTs of each center and all centres combined.

\begin{tabular}{|c|c|c|c|c|c|c|c|}
\hline & All CECTs & Center B & Center C & Center D & Center E & Center F & Center G \\
\hline No of CECTs & 388 & 49 & 74 & 59 & 73 & 63 & 69 \\
\hline No of CECTs performed per patient & $1.4(1-5)$ & $1.2(1-3)$ & $1.5(1-5)$ & $1.2(1-3)$ & $1.5(1-4)$ & $1.3(1-4)$ & $1.4(1-4)$ \\
\hline \multirow[t]{2}{*}{ Time to CECT } & $7(0-90)$ & $6(0-26)$ & $6.5(0-90)$ & $9(1-31)$ & $6(0-87)$ & $12(1-67)$ & $4(1-88)$ \\
\hline & IQR 3-13 & IQR 3-8 & IQR 3-16 & IQR 4-13 & IQR 3-11 & IQR 3-41 & IQR 2-11 \\
\hline \multicolumn{8}{|l|}{ Type of pancreatitis and time to CECT } \\
\hline \multirow[t]{2}{*}{ Oedematous pancreatitis } & $3(0-73)$ & $4(0-19)$ & $5.5(0-73)$ & $5(1-9)$ & $2(0-57)$ & $4(1-65)$ & $3(1-50)$ \\
\hline & IQR 2-7 & IQR $1-7$ & IQR 3-9 & IQR 2-8 & IQR $1-6$ & IQR 3-7 & IQR 2-4 \\
\hline \multirow[t]{2}{*}{ Necrotising pancreatitis } & $10(1-90)$ & $8(0-26)$ & $13(0-90)$ & $9(1-32)$ & $8(0-87)$ & $23(1-67)$ & $9(2-89)$ \\
\hline & IQR 5-21 & IQR 6-12 & IQR 6-33 & IQR 5-14 & IQR 4-15 & IQR $11-46$ & IQR 5-27 \\
\hline \multirow[t]{2}{*}{ Indeterminate pancreatitis } & $3(0-14)$ & $2(0-8)$ & $4(1-5)$ & $4(1-14)$ & $4(1-7)$ & $2(2-11)$ & $2(1-5)$ \\
\hline & IQR 2-5 & IQR $1-8$ & IQR 2-4 & IQR 2-5 & IQR $1-6$ & IQR $2-10$ & IQR $1-4$ \\
\hline
\end{tabular}

No=Number.

$\mathrm{IQR}=$ Inter quartile range.

Continuous variables are median. Range and IQR are displayed if applicable.

Time to CECT=Time from symptom onset to CECT in days.

Type of pancreatitis and time to CECT = Time from symptom onset to CECT in days divided by type of pancreatitis.

Table 3

Center independent and dependent kappa values for all categories scored.

\begin{tabular}{|c|c|c|c|c|c|c|c|}
\hline Category & All centres & Center B & Center C & Center D & Center E & Center F & Center G \\
\hline Type of pancreatitis & 0.370 & 0.317 & 0.342 & 0.309 & $0.098^{\mathrm{b}}$ & $0.838^{\mathrm{a}}$ & 0.360 \\
\hline Parenchymal Necrosis & 0.539 & 0.380 & $0.319^{\mathrm{b}}$ & 0.609 & $0.731^{\mathrm{a}}$ & 0.663 & 0.465 \\
\hline Necrosis - Head & 0.516 & 0.669 & 0.345 & $0.323^{\mathrm{b}}$ & $1.00^{\mathrm{a}}$ & 0.660 & 0.646 \\
\hline Necrosis - Neck & 0.618 & $0.922^{\mathrm{a}}$ & 0.577 & 0.822 & 0.660 & 0.364 & $0.236^{\mathrm{b}}$ \\
\hline Necrosis - Body & 0.628 & 0.766 & 0.611 & 0.687 & 0.873 & $0.392^{\mathrm{b}}$ & 0.570 \\
\hline Necrosis - Tail & 0.617 & 0.451 & 0.626 & 0.687 & $0.409^{\mathrm{b}}$ & $0.806^{\mathrm{a}}$ & 0.532 \\
\hline Extrapancreatic Necrosis & 0.326 & 0.321 & 0.504 & 0.293 & 0.120 & $0.877^{\mathrm{a}}$ & $0.024^{\mathrm{b}}$ \\
\hline Collections & 0.756 & 0.780 & 0.750 & $0.624^{\mathrm{b}}$ & $0.864^{\mathrm{a}}$ & 0.827 & 0.625 \\
\hline Location of Collections & 0.633 & 0.728 & $0.761^{\mathrm{a}}$ & 0.508 & 0.694 & 0.604 & $0.439^{\mathrm{b}}$ \\
\hline Characteristics of collections & 0.408 & 0.397 & 0.485 & 0.293 & 0.305 & $0.744^{\mathrm{a}}$ & $0.251^{\mathrm{b}}$ \\
\hline Wall & 0.675 & 0.638 & 0.638 & $0.588^{\mathrm{b}}$ & $0.777^{\mathrm{a}}$ & 0.726 & 0.632 \\
\hline Intraluminal gas/fluid level & 0.764 & 0.774 & $0.671^{\mathrm{b}}$ & 0.764 & $0.887^{\mathrm{a}}$ & 0.837 & 0.675 \\
\hline Collection - most appropriate term & 0.356 & 0.385 & 0.480 & $0.136^{\mathrm{b}}$ & 0.218 & $0.673^{\mathrm{a}}$ & 0.261 \\
\hline
\end{tabular}

a Highest kappa value for center B to G for each category.

b Lowest kappa value for center B to G for each category.

Table 4

Number of extrapancreatic necrosis (EXPN) scored by the local radiologists and central expert.

\begin{tabular}{|c|c|c|c|c|c|c|}
\hline & \multicolumn{2}{|c|}{ Total number of EXPN } & \multicolumn{2}{|c|}{ Combined necrosis } & \multicolumn{2}{|c|}{ Isolated EXPN } \\
\hline & Local rad & Central exp & Local rad & Central exp & Local rad & Central exp \\
\hline Yes & $126(33 \%)$ & $230(59 \%)$ & $85(22 \%)$ & $92(24 \%)$ & $41(11 \%)$ & $138(36 \%)$ \\
\hline No & $245(63 \%)$ & $110(28 \%)$ & $303(78 \%)$ & $296(76 \%)$ & $347(89 \%)$ & $250(64 \%)$ \\
\hline Indet & $17(4 \%)$ & $48(12 \%)$ & & & & \\
\hline
\end{tabular}

Total number of EXPN = diagnosis of EXPN (yes/no) and indeterminate by local radiologists and central expert,respectively.

Combined necrosis = Extrapancreatic necrosis with Parenchymal necrosis in cases where presence of EXPN (yes/no) was determined.

Isolated EXPN = Extrapancreatic necrosis without Parenchymal Necrosis in cases where presence of EXPN (yes/no) was determined.

Local rad $=$ Local radiologists.

Central exp $=$ Central expert radiologist.

Indet $=$ Indeterminate (radiologist was not able to determine the presence of EXPN, yes/no).

consistency in the investigation and reporting of data in clinical research [1]. However, the degree of interobserver agreement in the interpretation of CT findings using these new RAC criteria has been questioned [6-8].

Main findings of our study are twofold: on the one hand, the morphologic assessment of the RAC generates overall moderate to good interobserver agreement (range 0.516-0.764) among European radiologists in 9 out of 13 items evaluated. Importantly, agreement among raters was good in evaluating clinically important CT findings in patients with AP, such as the presence of parenchymal necrosis and gas bubbles. On the other hand, only fair agreement (range $0.326-0.408$ ) was obtained for items pertaining to necrosis of extrapancreatic tissues. The central expert diagnosed
EXPN significantly more frequent than the local radiologists (59\% versus $33 \%, P<0.0001$ ) with better correlation with patient outcome. Our findings suggest that radiologists are largely unfamiliar with the newly defined entity of EXPN.

Several explanations exist for the fair agreement in diagnosing EXPN and for characterisation of pancreatic collections on CT. The RAC regards $C T$ as the first-line imaging modality in AP, albeit acknowledging the fact that necrotic material within pancreatic collections is often overlooked [1]. It is well-established that ultrasound and magnetic resonance imaging are better capable of delineating the exact composition of pancreatic collections, especially for depicting necrotic material $[10,11]$. Furthermore, the CT diagnosis of EXPN and associated necrotic collections relies 
Table 5

Correlation of morphologic findings with clinical outcome by central and local radiologists.

\begin{tabular}{|c|c|c|c|c|c|c|c|}
\hline & \multirow[t]{2}{*}{ Total (N) } & \multicolumn{3}{|l|}{ IEP } & \multicolumn{3}{|c|}{ Isolated EXPN } \\
\hline & & Local & Central & P-value & Local & Central & $P$-value \\
\hline All cases & $(388)$ & 199 & 107 & 0.0001 & 41 & 138 & 0.0001 \\
\hline \multicolumn{8}{|l|}{ Organ Failure } \\
\hline Persistent & $(50)$ & $20(10.0 \%)$ & $5(4.7 \%)$ & 0.0001 & $4(9.7 \%)$ & $24(17.4 \%)$ & 0.0001 \\
\hline Transient & (51) & $15(7.5 \%)$ & $5(4.7 \%)$ & 0.002 & $4(9.7 \%)$ & 20 (14.5\%) & 0.0001 \\
\hline Mortality & (20) & $9(4.5 \%)$ & $4(3.7 \%)$ & 0.025 & $1(2.4 \%)$ & $7(5.1 \%)$ & 0.025 \\
\hline Intervention & (79) & $11(5.5 \%)$ & $6(5.6 \%)$ & $n s$ & $12(29.3 \%)$ & $26(18.8 \%)$ & 0.001 \\
\hline CRP (mg/l) & & 271 & 165 & 0.0001 & 298 & 318 & $n s$ \\
\hline SIRS & (104) & $41(20.6 \%)$ & $12(11.2 \%)$ & 0.0001 & $9(22.0 \%)$ & $38(27.5 \%)$ & 0.0001 \\
\hline
\end{tabular}

Cases scored as 'indeterminate' are not accounted for.

IEP = Interstitial Edematous Pancreatitis.

Isolated EXPN = Extrapancreatic Necrosis without Parenchymal Necrosis.

Local $=$ Local radiologists.

Central $=$ Central Expert.

$\mathrm{CRP}=\mathrm{C}$-reactive protein, highest value.

SIRS $=$ Systemic Inflammatory Response Syndrome

primarily on subjective secondary findings, such as 'heterogeneity' or the detection of various densities (liquid and non-liquid) within collections, rather than using the more objective and reproducible criteria of perfusion characteristics, which is used for detecting pancreatic parenchymal necrosis. Perfusion characteristics, however, cannot be used to diagnose EXPN because normal extrapancreatic fat does not enhance. In addition, the RAC acknowledges that EXPN is difficult to diagnose initially but becomes easier when the disease process evolves over time [1]. This is in line with our results with improved kappa values for EXPN diagnosis two weeks after symptom onset. Finally, reader expertise and familiarity seem equally important for diagnosing EXPN exemplified by the excellent interobserver agreement between the central expert and the local radiologist affiliated with center $\mathrm{F}$ that admits the highest number of patients with AP annually.

In a previous study on CT assessment of morphologic features of AP, good to excellent interobserver agreement was found in 55 cases of AP [12]. However, this study used selected cases biased towards severe disease (likely associated with more established peripancreatic collections) reviewed by tertiary experts. Although imaging is rarely required for mild AP, most patients who undergo CT for evaluation of AP turn out to have interstitial pancreatitis. Our study more closely resembles clinical practice in different European countries by enrolling patients with AP consecutively and evaluating unselected CECTs encompassing the full spectrum of morphologic abnormalities in AP, including mild and equivocal cases.

Previous studies show considerable differences in clinical outcome, treatment strategies, and prognoses between the various morphological types of AP [13-17]. Clinical outcome of EXPN is worse compared with acute interstitial pancreatitis, but better than pancreatic necrosis $[13,15,17]$. Patients with EXPN stay in hospital considerable longer, develop more often organ failure, and undergo interventional therapy significantly more frequent than those with interstitial pancreatitis. Moreover, when infection ensues of necrotic collections in EXPN patients, outcome, therapy, and prognosis are similar to those with infected pancreatic necrosis [15]. In our study, the interpretation by the central expert more closely corroborated with actual clinical outcome. As such, accurate differentiation between the types of AP is important both from a clinical perspective as for consistent reporting of research and reliable comparison of inter-institutional data.

This study has some limitations. First, a single central expert radiologist served as standard of reference, potentially introducing bias. We considered this a limited risk because of his extensive experience in pancreatic imaging, his involvement in the development of the RAC, the superior correlation with patient outcome, and the excellent agreement with a local radiologist with

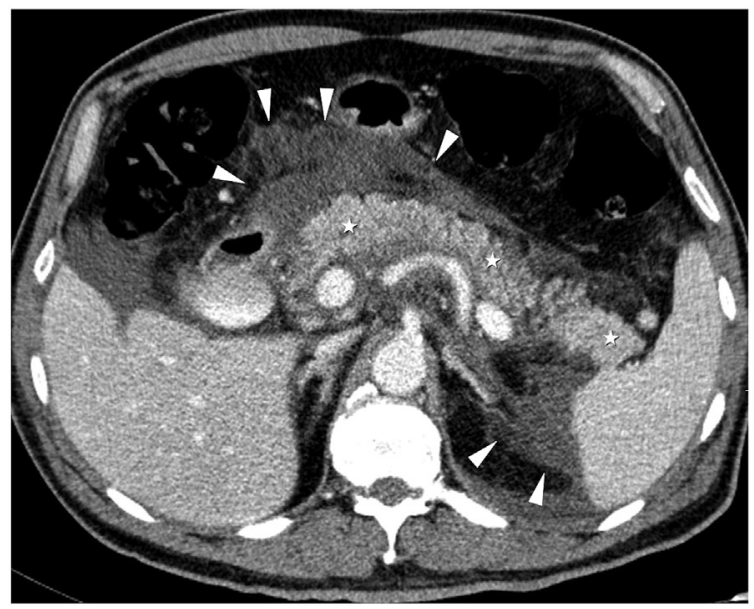

a

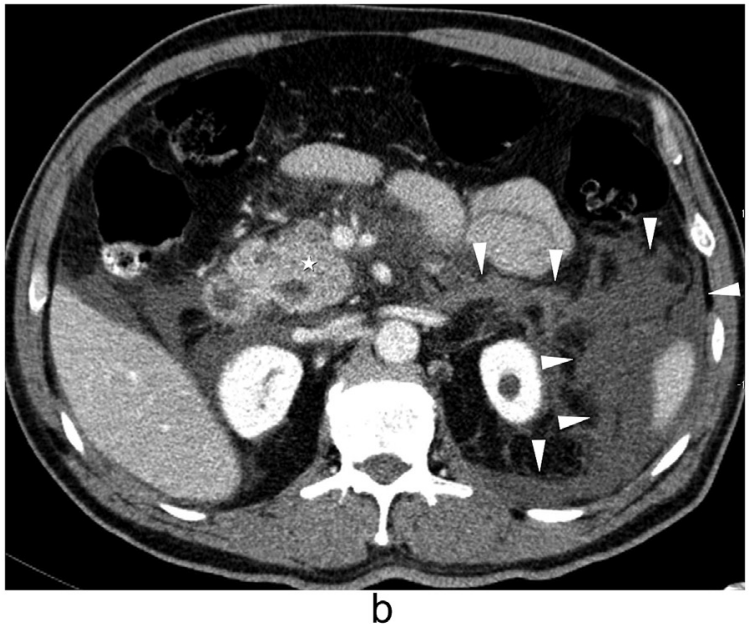

Fig. 1. 70-year-old male with acute pancreatitis (a, b). The pancreas enhances heterogeneously (asterisks) but no apparent necrosis was observed by both reviewers. Peripancreatic collections are present in the retroperitoneal pancreatic compartment and transverse mesocolon (arrowheads pointing at the borders). The local reviewer scored this as interstitial pancreatitis with acute peripancreatic fluid collections; the central reviewer as necrotising pancreatitis, subtype EXPN, with acute necrotic collections. 


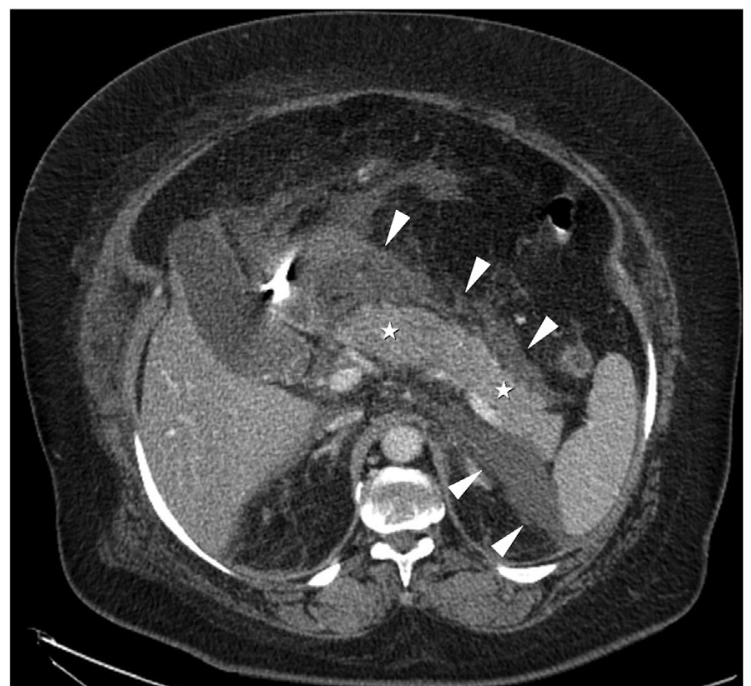

a

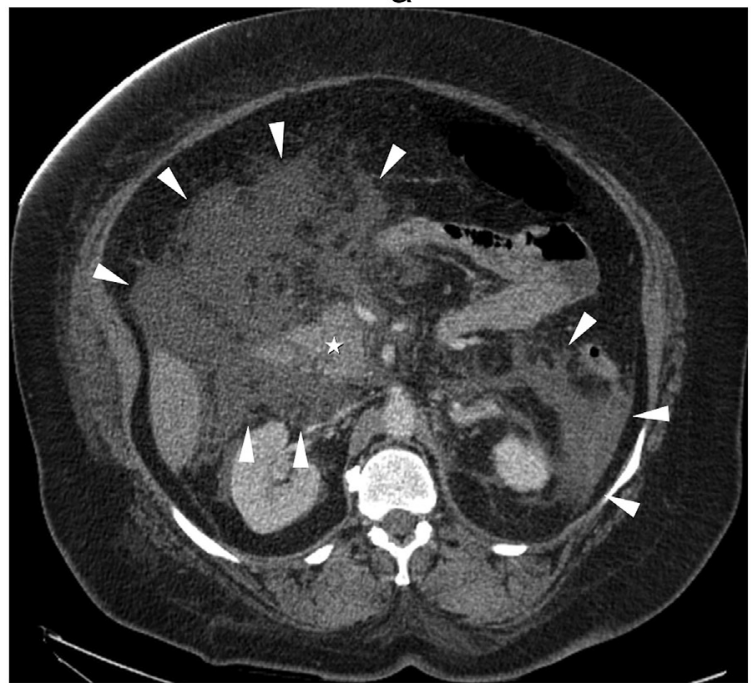

b

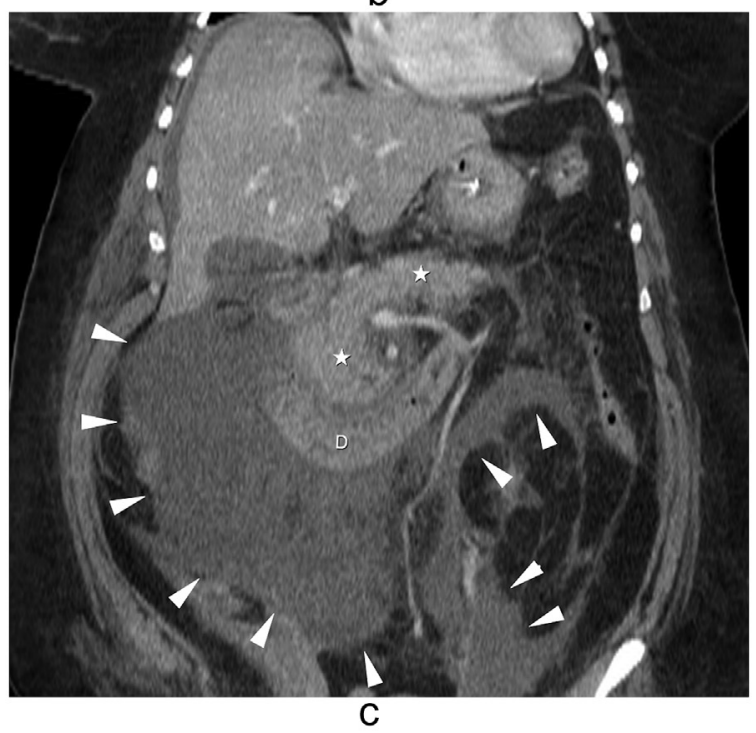

Fig. 2. 55-year-old female with acute pancreatitis $(a-c)$. Normal enhancement of pancreatic parenchyma was noted by both reviewers (asterisks). Extensive peripancreatic collections are present in the retroperitoneum bilaterally and transverse mesocolon (arrowheads pointing at the borders). These represented 'acute peripancreatic fluid collections' and 'acute necrotic collections' according to the local and central reviewer, respectively. Coronal reformatted image (c) depicts the magnitude of collections. similar expertise. Second, given the retrospective design of this study, we did not investigate to what extent the inconsistencies observed eventually affected clinical decision-making. Future studies should focus on this interesting topic. Finally, we merely studied the interobserver agreement of morphological abnormalities in AP and did not correlate imaging findings with histopathology.

Results of this study have revealed areas of controversy when using the RAC criteria for CT assessment, especially pertaining to distinguishing interstitial pancreatitis from EXPN only. There are several options for improving consistent reporting in AP. Both radiologists and clinicians need to become better familiar with imaging features of EXPN (i.e. by education or training). Second, the definition of EXPN should preferably be redefined such that stronger interrater agreement will be achieved, even among readers with varying expertise. For example, by adding a time interval of 2 weeks before its diagnosis or by using an alternative imaging (MRI or US) modality as these are better capable of detecting necrotic material within collections [10]. Third, a greater role should be attributed to MRI for overall evaluation of AP. Finally, as has been alluded to in previous reports, a three-degree morphologic classification system ('interstitial pancreatitis' refers to normal enhancing parenchyma without collections, 'EXPN' refers to normal perfused pancreatic parenchyma with pancreatic collections, and 'necrotising pancreatitis' refers to parenchymal necrosis with or without associated collections) could potentially lead to less interobserver variability as the differentiation between the various types of pancreatic collections becomes less of an issue $[18,19]$. Additionally, Such a system would likely be more in concordance with clinical grades of severity [13-17,20].

In conclusion, this study found only moderate interrater agreement for identification of EXPN. For correctly identifying EXPN and necrotic collections on CT, a diligent search for heterogeneity within pancreatic collections is crucial for accurate and consistent reporting of imaging findings (see Figs. 1 and 2).

\section{Disclosure}

The authors declare no conflict of interests.

\section{Funding resources}

No funding was involved in any part of the study or manuscript.

\section{Acknowledgements}

The authors thank Luigi Santoro, Division of Epidemiology and Biostatistics, European Institute of Oncology, for assistance with statistical analysis. The group was part of Pancreas $2000-$ a research program for future pancreatologists, who in part also supported this study (www.pancreas2000.org).

\section{Appendix A. Supplementary data}

Supplementary data related to this article can be found at http:// dx.doi.org/10.1016/j.pan.2016.08.007.

\section{References}

[1] Banks PA, Bollen TL, Dervenis C, Gooszen HG, Johnson CD, Sarr MG, et al. Classification of acute pancreatitis-2012: revision of the atlanta classification and definitions by international consensus. Gut 2013;62:102-11.

[2] Bradley 3rd EL. A clinically based classification system for acute pancreatitis Summary of the international symposium on acute pancreatitis, atlanta, ga, september 11 through 13, 1992. Arch Surg 1993;(128):586-90.

[3] Bollen TL. Imaging of acute pancreatitis: update of the revised atlanta 
classification. Radiol Clin North Am 2012;50:429-45.

[4] Thoeni RF. The revised Atlanta classification of acute pancreatitis: its importance for the radiologist and its effect on treatment. Radiology 2012;262:751-64.

[5] Shyu JY, Sainani NI, Sahni VA, Chick JF, Chauhan NR, Conwell DL, et al. Necrotizing pancreatitis: diagnosis, imaging and intervention. Radiographics 2014;34:1218-39.

[6] Windsor JA, Petrov MS. Acute pancreatitis reclassified. Gut 2013;62:4-5.

[7] Revision of the atlanta classification of acute pancreatitis: the editorial perspectiveGress TM, El-Omar EM, editors. Gut 2013;62:1.

[8] Lerch MM. Classifying an unpredictable disease: the revised atlanta classification of acute pancreatitis. Gut 2013;62:2-3.

[9] Landis JR, Koch GG. The measurement of observer agreement for categorical data. Biometrics 1977:33:159-74.

[10] Morgan DE, Baron TH, Smith JK, Robbin ML, Kenney PJ. Pancreatic fluid collections prior to intervention: evaluation with $\mathrm{mr}$ imaging compared with $\mathrm{ct}$ and us. Radiology 1997;203:773-8.

[11] Balthazar EJ. Acute pancreatitis: assessment of severity with clinical and ct evaluation. Radiology 2002;223:603-13.

[12] van Santvoort HC, Bollen TL, Besselink MG, Banks PA, Boermeester MA, van Eijck $\mathrm{CH}$, et al. Describing peripancreatic collections in severe acute pancreatitis using morphologic terms: an international interobserver agreement study. Pancreatology 2008;8:593-9.

[13] Singh VK, Bollen TL, Wu BU, Repas K, Maurer R, Yu S, et al. An assessment of the severity of interstitial pancreatitis. Clin Gastroenterol Hepatol 2011;9: 1098-103.

[14] Sakorafas GH, Tsiotos GG, Sarr MG. Extrapancreatic necrotizing pancreatitis with viable pancreas: a previously under-appreciated entity. J Am Coll Surg 1999; 188:643-8.

[15] Bakker OJ, van Santvoort H, Besselink MG, Boermeester MA, van Eijck C, Dejong K, et al. Extrapancreatic necrosis without pancreatic parenchymal necrosis: a separate entity in necrotising pancreatitis? Gut 2013;62:1475-80.

[16] Rana SS, Sharma V, Sharma RK, Chhabra P, Gupta R, Bhasin DK. Clinical significance of presence and extent of extrapancreatic necrosis in acute pancreatitis. J Gastroenterol Hepatol 2015;30:794-8.

[17] Wang M, Wei A, Guo Q, Zhang Z, Lu H, Li A, et al. Clinical outcomes of combined necrotizing pancreatitis versus extrapancreatic necrosis alone. Pancreatology 2016;16:57-65.

[18] Bollen TL, Besselink MG, van Santvoort HC, Gooszen HG, van Leeuwen MS. Toward an update of the atlanta classification on acute pancreatitis: review of new and abandoned terms. Pancreas 2007;35:107-13.

[19] Bollen TL, van Santvoort HC, Besselink MG, van Leeuwen MS, Horvath KD, Freeny PC, et al. The atlanta classification of acute pancreatitis revisited. $\mathrm{Br} J$ Surg 2008;95:6-21.

[20] Lankisch PG, Struckmann K, Lehnick D. Presence and extent of extrapancreatic fluid collections are indicators of severe acute pancreatitis. Int J Pancreatol 1999:26:131-6. 